\title{
A comparative study on unconstrained hand biometrics
}

\author{
Alberto de Santos Sierra \\ Carmen Sánchez Ávila \\ Group of Biometrics, Biosignals and Security \\ Universidad Politécnica de Madrid \\ Campus de Montegancedo \\ \{alberto, csa\}@cedint.upm.es
}

\author{
Javier Guerra Casanova \\ Gonzalo Bailador del Pozo \\ Group of Biometrics, Biosignals and Security \\ Universidad Politécnica de Madrid \\ Campus de Montegancedo \\ \{jguerra, gbailador\}ecedint.upm.es
}

\begin{abstract}
Biometrics applied to mobile devices are of great interest for security applications. Daily scenarios can benefit of a combination of both the most secure systems and most simple and extended devices.

This document presents a hand biometric system oriented to mobile devices, proposing a non-intrusive, contact-less acquisition process where final users should take a picture of their hand in free-space with a mobile device without removals of rings, bracelets or watches.

The main contribution of this paper is threefold: firstly, a feature extraction method is proposed, providing invariant hand measurements to previous changes; second contribution consists of providing a template creation based on hand geometric distances, requiring information from only one individual, without considering data from the rest of individuals within the database; finally, a proposal for template matching is proposed, minimizing the intra-class similarity and maximizing the inter-class likeliness. The proposed method is evaluated using three publicly available contact-less, platform-free databases. In addition, the results obtained with these databases will be compared to the results provided by two competitive pattern recognition techniques, namely Support Vector Machines (SVM) and $k$-Nearest Neighbour, often employed within the literature.

Therefore, this approach provides an appropriate solution to adapt hand biometrics to mobile devices, with an accurate results and a non-intrusive acquisition procedure which increases the overall acceptance from the final user.
\end{abstract}

Keywords: Biometrics, hand geometry, mobile devices, support vector machine, security

\section{INTRODUCTION}

New trends in biometrics are inclined to adapt both identification and verification process to mobile devices in order to provide real scenarios and applications with a more secure frame. In fact, upcoming applications related to electronic commerce are demanding more trustworthy and reliable techniques to ensure their operations and transactions [1], for instance. In other words, biometrics are requested to provide an appropriate alternative to current pin codes and passwords.

Furthermore, commercial biometric systems normally have no constraints in terms of computational cost or involved hardware but they do aim the highest accuracy in personal identification. In contrast, applying biometrics to mobile devices requires a reconsideration of previous lack of constraints since a mobile device is at present far from being comparable to current biometric systems in terms of hardware.

Based on these concerns, this document presents a biometric system based on hand geometry oriented to mobile devices, since hand images were acquired with mobile devices. This approach offers the possibility of identifying individuals easily with a non-intrusive acquisition procedure, using a picture taken with the mobile phone and avoiding the use of a flat surface to place the hand, providing this system with a noncontact characteristic. Moreover, the hand can be acquired without constraints in orientation, distance to camera or illumination, since the proposed technique within this paper is invariant to previous changes. This property provides an increase in the acceptance of the biometric technique by the final user, together with the fact that no removal of rings, watches and the like is required for image acquisition.

In contrast, such lack of constraints in acquisition demands a more challenging solution in relation to segmentation and feature extraction. The former operation must be able to isolate completely hand from background, regardless what is behind the hand. In case of feature extraction, the template must be independent from which hand is considered for identification (left or right hand) and invariant to changes in orientation, position, distance to camera and the like. In addition, the proposed template considers finger widths and lengths and, besides, information from four fingers (index, middle, ring and little/pinky) is considered, instead of global features from the whole hand.

The proposed method is evaluated using three publicly available contact-less, platform-free databases. In addition, the results obtained with these databases will be compared to the results provided by two competitive pattern recognition techniques, namely Support Vector Machines (SVM) and $k$ Nearest Neighbour, often employed within the literature.

The layout of the paper is arranged as follows: Section II provides a literature review in hand biometrics, drawing attention to hand geometry approaches. Section III describes both the segmentation procedure and how features are extracted. Before presenting the results in Section V, a description of the databases involved to evaluate the biometric system is provided in Section IV. Finally, this document ends with conclusions 
and future work in Section VI.

\section{RELATED WORK}

Hand biometric systems have evolved from early approaches which considered flat-surface and pegs to guide the placement of the user's hand [2], [3], [4], to completely platform-free, non-contact techniques were user collaboration is almost not required [5], [6], [7], [8]. This development can be classified into three categories according to the image acquisition criteria [9]:

- Constrained and contact based. Systems requiring a flat platform and pegs or pins to restrict hand degree of freedom [3], [4].

- Unconstrained and contact based. Peg-free scenarios, although still requiring a platform to place the hand, like a scanner [7], [10].

- Unconstrained and contact-free. Platform-free and contactless scenarios where neither pegs nor platform are required for hand image acquisition [6], [11].

An aspect of relevance regards how the hand is acquired concerning not only the acquisition devices but also to what extent hand background is under control.

For the sake of a precise acquisition, hand is usually located on a flat surface provided with guiding pegs ensuring that hand is exactly placed on the same position. However, some problems arise from this approach which concern shape deformation, an increase in the device acquisition complexity and, more recently, contact-based acquisition devices can be considered controversial regarding hygiene and public-health issues [6].

Regarding invasiveness, most of previous works require a removal of rings, bracelets and similar complements [12], although many trends tend to extract hand features and descriptors without requiring any removal [13], [11].

Hand biometric acceptation was assessed in [14], [15], [12] evaluating the performance of the biometric system in relation to the number of attempts in accessing the system. In fact, the repeated used of the device provides an increase in the identification accuracy of participants.

New trends in biometrics tend to adapt current systems to mobile devices. However, not every biometric technique is suitable for this adaptation. Furthermore, mobile devices imply certain limitations in relation to computational cost and performance efficiency and accuracy. Obviously, mobile security is not so demanding as, for instance, an application in an international airport. In the literature, there exist previous approaches concerning biometrics and mobiles involving different biometric characteristics: Face Recognition on Symbian OS [16], [17], Voice Recognition [18], Keystroke Dynamics [19], Hand [11], Palmprint [20] or Finger Pressure [18], [19], [21]. All previous work coincide on the same conclusions: mobile devices imply limitations for biometric accuracy and efficiency, but provide a high degree of security in daily applications.

Finally, an overview on recent hand biometrics systems is presented in Table II. This table presents the relation between the features required for identification, the method proposed, the population involved together with the results obtained, in terms of Equal Error Rate (EER).

\section{Methodology}

Thisi section describes the proposed method in detail concerning the segmentation, feature extraction and template creation and matching.

\section{A. Segmentation}

The proposed segmentation algorithm is based on multiscale aggregation [31], [32]. Concretely, the method considers image $I$ as a graph $G=(V, E, W)$, where nodes $v_{i} \in V$ correspond to pixels in image; edges $e_{i, j} \in E$ represent the union between two nodes $v_{i}$ and $v_{j}$; weights $w_{i, j} \in W$ describe the similarity between two nodes $v_{i}$ and $v_{j}$ associated by an edge $e_{i, j}$.

The main contribution of this algorithm is to describe each node as a similarity function based on a specific neighbourhood. In other words, each node $v_{i}$ is described as a function $\phi_{v_{i}}$, assuming a normal distribution $\mathcal{N}(\mu, \sigma)$ in terms of intensities within the 4-neighbour structure. Parameters $\mu$ and $\sigma$ make reference to the average and standard deviation of the intensity in the proposed neighbour structure.

Therefore, the weight $w_{i, j}$ is defined in terms of functions $\phi_{v_{i}}$ and $\phi_{v_{j}}$ as in Equation 1:

$$
w_{i, j}=\int_{\alpha} \sqrt{\phi_{v_{i}} \phi_{v_{j}}} d \alpha
$$

where $\alpha$ represents the color space. In this case, this color space corresponds to CIELAB, concretely the $a$ layer.

The method carries out the following procedure until only two segment remains:

- Obtain set graph $G$ for image $I$

- Order pair of nodes according to weights $W$

- Aggregate nodes in descendent order, based on previous ordering in $W$

- Calculate function $\phi$ for each aggregated segment.

- Provide neighbour structure applying Delaunay triangulation

Finally, this method comes out with precise and accurate results for hand segmentation [32]. The result section shows that the application of this method together with the feature extraction provides independency against blur effect.

\section{B. Feature Extraction and Template Creation}

This section defines the features to be extracted from hand in order to reduce the biometric information contained within the hand to more comparable and measurable parameters. These features must describe and define the hand uniquely and univocally, and must remain invariant to changes of size, distance to camera, rotation and similar variations in acquisition. Some previous works provide similar templates based on width fingers and distances extracted from hand [33], [34], and others consider free-space acquisition [10], [6], but without considering a high degree of freedom in hand changes and mobile devices acquisition. 


\begin{tabular}{|c|c|c|c|c|c|}
\hline Year & Ref. & Features & Method & $\begin{array}{l}\text { Population } \\
\text { Size }\end{array}$ & $\overline{\operatorname{EER}(\%)}$ \\
\hline \multirow[t]{4}{*}{2007} & [6] & 5-35 distances & Projective invariants & 23 & 2.11 \\
\hline & [22] & 23 distances & $\begin{array}{l}\text { Entropy Discretization and } \\
\text { SVM }\end{array}$ & 100 & 5 \\
\hline & {$[5]$} & 15 hand distances & SVM & 18 & 8 \\
\hline & [23] & 5 distances & AAM & 18 & 5 \\
\hline \multirow[t]{3}{*}{2008} & [24] & 30-40 finger widths & SVM & $20-30$ & $4.2-6.3$ \\
\hline & [25] & 15 graph distances & DBNN & 250 & 0.89 \\
\hline & [26] & Palmprint & Gabor Filters and SVM & 49 & 1.7 \\
\hline \multirow[t]{4}{*}{2009} & {$[8]$} & Zernike Descriptors & Fusion SVDD & 86 & 1.5 \\
\hline & [27] & $2 \mathrm{D}$ and $3 \mathrm{D}$ features & Savitzky-Golay filters & 177 & 2.6 \\
\hline & [11] & Contour & DTW alignment & 45 & 3.7 \\
\hline & [28] & 40 distances & SVM & 260 & $\begin{array}{l}0.0035- \\
5.7\end{array}$ \\
\hline \multirow[t]{2}{*}{2010} & [29] & 30 distances and angles & Correlation & 50 & 4.2 \\
\hline & [30] & $\begin{array}{l}\text { 2D and 3D palmprint and } \\
\text { geometry }\end{array}$ & Surface Code & 114 & 0.71 \\
\hline
\end{tabular}

TABLE I

LITERATURE REVIEW ON MOST RECENT WORKS RELATED TO CONTACTLESS HAND BIOMETRICS BASED ON HAND GEOMETRY. THIS TABLE PRESENTS THE RELATION BETWEEN THE FEATURES REQUIRED FOR IDENTIFICATION, THE METHOD PROPOSED, THE POPULATION INVOLVED TOGETHER WITH THE RESULTS OBTAINED, IN TERMS OF EQUAL ERROR RATE (EER).

After segmentation, tips an valleys within hand are detected [24] in order to established some landmarks points from which geometric features can be extracted.

The feature extraction presented in this method consists of the following steps:

1) Divide the finger from the basis to the tip in $m$ parts and obtain the widths corresponding to each division.

2) Form $n$ groups from previous set of geometrical features, by averaging $\left\lfloor\frac{m}{n}\right\rfloor$ elements in each group.

3) Create set $\Delta_{f_{k}}=\frac{\left\{\delta_{1}^{f_{k}}, \ldots, \delta_{n}^{f_{k}}\right\}}{\delta_{f_{k}}}$ where each $\delta_{t}^{f_{k}}$ is defined as the average value of the $t$-th set of elements $\left\lfloor\frac{m}{n}\right\rfloor$, for each finger $f_{k} \in\left\{f_{\text {index }}, f_{\text {middle }}, f_{\text {ring }}, f_{\text {little }}\right\}$. Reader may notice that set $\Delta_{f_{k}}$ is normalized for each finger.

4) Template $\Delta$ contains every set $\Delta_{f_{k}}$ for each finger, with a total of $M=4 \times n$ elements per template.

This steps has described how the distances are extracted and how the template is created for each hand. However, the user template contains more information so that the posterior identification and verification is more precise.

The template is created by considering a total of $N$ samples of an individual. Each sample is a vector of $M$ components extracted form a hand acquisition. The method creates in addition two parameters associated to the template: a vector $\mu_{\text {template }}$ as the average of each element along the $N$ samples; a deviation factor, $\sigma_{\text {template }}$, indicating the deviation of the differences between every pair of samples. In other words, since there are $N$ samples for template creation, a total of $\left(\begin{array}{l}N \\ 2\end{array}\right)$ combinations taking pairs of vectors. From this vector, the euclidean difference is computed and the standard deviation of all vectors is calculated and gathered under $\sigma_{\text {template }}$.
In addition, two more parameters are defined: vector $\pi$ and number $\gamma$. The former vector states which elements within template are less invariant along the $N$ samples. The latter number is defined as in Equation 2:

$$
\gamma=\frac{1}{M}\left(\frac{\mu_{\text {template }}}{\sigma_{\text {template }}} \pi^{T}\right)
$$

This number is related to the coefficient of variation of those samples which are less invariant within the $N$ samples.

Finally, the template $\mathcal{H}$ is created as $\mathcal{H}=$ $\left(\mu_{\text {template }}, \sigma_{\text {template }}, \pi, \sigma\right)$.

\section{Template matching}

One of the main contribution of this paper consists on the template matching. This template comparison is proposed based on a template $\mathcal{H}$ and a certain sample $h$, which can belong to the class associated to $\mathcal{H}$ or to other different class.

Therefore, the probability that sample $h$ belongs to $\mathcal{H}$ is defined by $p(h \mid \mathcal{H})$ in Equation 3:

$$
p(h \mid \mathcal{H})=\frac{1}{M} e^{-a H H^{T}}
$$

where $H$ is defined as in Equation 4

$$
H=\frac{1}{\gamma}\left(\frac{h-\mu_{\text {template }}}{\sigma_{\text {template }}} \circ \pi\right)
$$

where operator $A \circ B=\left[a_{i j} b_{i j}\right]_{\forall i, j}$ is defined as the Hadamard product, an entrywise multiplication for any two matrices $A, B$.

This probability $p(h \mid \mathcal{H})$ is within the interval $[0,1]$, indicating that sample $h$ belongs to user with template $\mathcal{H}$ as 
$p(h \mid \mathcal{H}) \rightarrow 1$, and vice versa.

Therefore, the biometric verification based on this approach can be carried out by stating a threshold $t h \in[0,1]$, so that an individual (with template $\mathcal{H}_{k}$ ) accesses the system providing a sample $h_{k}$, then the user is correctly verified (authenticated) if $p\left(h_{k} \mid \mathcal{H}_{k}\right) \geq t h$. Otherwise, the user is rejected. Similarly, the identification is considered by considering same previous threshold $t h$, so that, provided a sample of a user, $h_{k}$, the system must decide whom the sample belongs to, or, whether the user is not enrolled in the system. In other words, if $\arg _{i}\left(\min p\left(h_{k} \mid \mathcal{H}_{i}\right) \geq t h\right)$ determines that $i=k$ then the sample $h_{k}$ is properly identified, otherwise the user is not enrolled in the system. Some approaches in literature fail in associating sample $h_{k}$ as a non-existing profile, since they provide the most likelihood an similar class, even if the sample provided by $h_{k}$ corresponds to a non-registered individual [26], [7].

\section{DATABASES}

The proposed schemes within this paper are evaluated considering three public databases.

The first database contains hand acquisitions of 120 different individuals of an age range from 16 to 60 years old, gathering males and females in similar proportion.

With the aim of a contact-less approach in hand biometrics, hand images were acquired without placing the hand on any flat surface neither requiring any removal of rings, bracelets or watches. Instead, the individual was required to open his/her hand naturally, so the mobile device (an HTC) could take a photo of the hand at $10-15 \mathrm{~cm}$ of distance with the palm facing the camera.

This acquisition procedure implies no severe constraints on neither illumination nor distance to mobile camera, being every acquisition carried out under natural light. In addition, it is a database with a huge variability in terms of size, skin color, orientation, hand openness and illumination conditions. In order to ensure a proper feature extraction, independently on segmentation, acquisitions were taken on a defined bluecoloured background, so that segmentation can be easily performed, focusing on hands. Both hands were taken, in a total of two sessions: During the first session, 10 acquisitions from both hands are collected; second session is carried out after 10-15 minutes, collecting again 10 images per hand. The image size provided by the device is $640 \times 340$ pixels. This first database is publicly available at www.gb2s.es. This database will be referred in this paper as GB2S database.

Second database is named 'IIT Delhi Palmprint Image Database version 1.0' [35], and it is a palmprint image database consisting of a hand images collection from the students and staff at IIT Delhi, New Delhi, India. This database has been acquired in the IIT Delhi campus during July 2006 Jun 2007 using a simple and touchless imaging setup. All the images are collected in the indoor environment and employ circular fluorescent illumination around the camera lens. The currently available database is from 235 users, all the images are in bitmap format. All the subjects in the database are in

\begin{tabular}{cccc}
\hline \hline & GB2S & IITDelhi & UST \\
\hline$k$-NN & $4.3 \pm 0.2$ & $3.9 \pm 0.2$ & $3 \pm 0.1$ \\
SVM & $3.1 \pm 0.1$ & $2.4 \pm 0.1$ & $2.1 \pm 0.2$ \\
Proposed & $2.5 \pm 0.2$ & $2 \pm 0.2$ & $1.4 \pm 0.1$ \\
\hline \hline
\end{tabular}

TABLE II

EQUAL ERROR RATE FOR EACH DATABASE AND METHOD. THE RESULTS OBTAINED WITH GB2S DATABASE ARE WORST IN COMPARISON TO THE OTHER DATABASES SINCE GB2S DATABASE PRESENT MORE VARIABILITY IN TERMS OF HAND ROTATION, DISTANCE TO CAMERA AND ENVIRONMENTAL CONDITIONS.

the age group 12-57 years. Seven images from each subject, from each of the left and right hand, are acquired in varying hand pose variations. Each of the subject is provided with live feedback to present his/her hand in the imaging region. The resolution of these images is $800 \times 600$ pixels. This database will be referred in this paper as IITDelhi database.

Third database acquisition setup is inherently simple and does not employ any special illumination nor does it make use of any pegs to cause any inconvenience to users. The Olympus C-3020 digital camera (1280 x 960 pixels) was used to acquire both images from 287 individuals, with ten samples per user. The users were only requested to make sure that their fingers do not touch each other and most of their hand (back side) touches the imaging table. A further explanation of this database can be found in [36]. This database will be referred in this paper as UST database.

\section{RESUlts}

Evaluation in hand biometrics involves assessing to what extent features are invariant to changes (position, scale or orientation) and the accuracy in identifying and verifying individuals given a database.

The proposed method will be compared in terms of technical evaluation [14] to two competitive pattern recognition techniques, namely Support Vector Machines (SVM) and $k$ Nearest Neighbour $(k$-NN) [22]. Although a wide explanation of these approaches is beyond the scope of this paper, some concerns must be taken into account with reference to the manner both approaches carry out classification. Both SVM and $k$-NN create a template based on information from other individuals, in contrast to the proposed template method, where only samples from a single individual are required to conform the template.

In addition, there exist another difference concerning the similarity score provided by these methods.

Table II presents the Equal Error Rates obtained for each method ( $k$-NN, SVM and proposed) in relation to the three employed databases in the evaluation (GB2S, IITDelhi and UST).

Table III gathers the results obtained applying the proposed method and standard width feature extraction [4], [3]. It shows that the use of this feature extraction method decreases the EER for each pattern recognition method, obtaining a remarkable improvement compared to standard extraction methods. 


\begin{tabular}{ccc}
\hline \hline & Standard Method [4], [3] & Proposed Method \\
\hline$k$-NN & $7.1 \pm 0.2$ & $4.3 \pm 0.2$ \\
SVM & $6.3 \pm 0.2$ & $3.1 \pm 0.1$ \\
Proposed & $4.8 \pm 0.1$ & $2.5 \pm 0.2$ \\
\hline \hline
\end{tabular}

TABLE III

COMPARATIVE STUDY OF THE IMPROVEMENT ACHIEVED BY THE PROPOSED FEATURE EXTRACTION METHOD FOR EACH PATTERN RECOGNITION METHOD (PROPOSED, $k$-NN AND SVM). THE IMPROVEMENT ACHIEVED BY THE PROPOSED METHOD IS REMARKABLE.

In addition, results presented in Table III where obtained by using the GB2S database. It is not difficult to assume that the feature extraction method conserves its properties, regardless the database.

Finally, the number of training samples was 4 and the number of feature extracted was also 20 per finger, as in all the evaluation scenarios.

\section{Vi. Conclusions And Future Work}

The contribution of this paper is threefold: Firstly, it proposes a feature extraction method, invariant to different changes in hand image acquisition. In addition, it presents a template which requires data only from one single user, avoiding to use data from the rest of the users within the database. Finally, the template matching is able to minimize the intra-class similarity variation and maximize the inter-class likeliness, since this template matching only considers those positions within the template with less intra-class variation, instead of comparing the whole template.

In fact, the use of both the feature extraction method and the template creation decreases remarkably the Equal Error Rate of the system, regardless the database involved.

The evaluation was carried out with three publicly available contact-less, platform-free databases, comparing the results obtained to two competitive pattern recognition techniques, namely Support Vector Machines (SVM) and $k$ Nearest Neighbour, widely employed within the literature. The method proposed improves the results obtained with the former pattern recognition techniques in terms of identification and verification performance.

In general, the low computational cost required with this approach, together with the accurate performance in human identification makes of this proposed method a suitable scheme for devices with low hardware requirements, and its unconstrained and contact-less acquisition procedure can extend the applicability of this proposed system to a wide number of scenarios. In addition, there is no constraint on the quality of the camera during the acquisition, since one of the database was obtained with a mobile phone.

Considering future work, authors would like to provide fusion schemes for both hands, together with palmprint or knuckleprint. Furthermore, more comparative evaluations will be carried out concerning other public databases and competitive methods in literature. Finally, an adaptation to current biometric standards ISO/IEC JTC1/SC37 will be also considered.

\section{REFERENCES}

[1] Van Thanh, D. (2000) Security issues in mobile ecommerce. Database and Expert Systems Applications, 2000. Proceedings. 11th International Workshop on, pp. $412-425$.

[2] Golfarelli, M., Maio, D., and Malton, D. (1997) On the error-reject tradeoff in biometric verification systems. Pattern Analysis and Machine Intelligence, IEEE Transactions on, 19, 786 -796.

[3] Jain, A. and Duta, N. (1999) Deformable matching of hand shapes for user verification. Image Processing, 1999. ICIP 99. Proceedings. 1999 International Conference on, oct., pp. 857 -861 vol.2.

[4] Sanchez-Reillo, R., Sanchez-Avila, C., and Gonzalez-Marcos, A. (2000) Biometric identification through hand geometry measurements. Pattern Analysis and Machine Intelligence, IEEE Transactions on, 22, 1168 1171.

[5] Jiang, X., Xu, W., Sweeney, L., Li, Y., Gross, R., and Yurovsky, D. (2007) New directions in contact free hand recognition. Image Processing, 2007. ICIP 2007. IEEE International Conference on, 16 2007-oct. 19, pp. II -389 -II -392.

[6] Zheng, G., Wang, C.-J., and Boult, T. (2007) Application of projective invariants in hand geometry biometrics. Information Forensics and Security, IEEE Transactions on, 2, 758-768.

[7] Adán, M., Adán, A., Vázquez, A. S., and Torres, R. (2008) Biometric verification/identification based on hands natural layout. Image Vision Comput., 26, 451-465.

[8] Amayeh, G., Bebis, G., and Nicolescu, M. (2009) Improving handbased verification through online finger template update based on fused confidences. Biometrics: Theory, Applications, and Systems, 2009. BTAS '09. IEEE 3rd International Conference on, sept., pp. $1-6$.

[9] Kanhangad, V., Kumar, A., and Zhang, D. (2011) Contactless and pose invariant biometric identification using hand surface. Image Processing, IEEE Transactions on, 20, 1415 -1424.

[10] Ferrer, M., Fabregas, J., Faundez, M., Alonso, J., and Travieso, C. (2009) Hand geometry identification system performance. Security Technology, 2009. 43rd Annual 2009 International Carnahan Conference on, 5-8, pp. 167-171.

[11] de Santos Sierra, A., Guerra Casanova, J., Sánchez Ávila, C., and Jara Vera, V. (2009) Silhouette-based hand recognition on mobile devices. 43rd Annual 2009 International Carnahan Conference on Security Technology, 2009., oct., pp. 160-166.

[12] Kukula, E., Elliott, S., Gresock, B., and Dunning, N. (2007) Defining habituation using hand geometry. Automatic Identification Advanced Technologies, 2007 IEEE Workshop on, 7-8, pp. 242-246.

[13] Yoruk, E., Konukoglu, E., Sankur, B., and Darbon, J. (2006) Shapebased hand recognition. IEEE Transactions on Image Processing, 15, 1803-1815.

[14] Kukula, E. and Elliott, S. (2005) Implementation of hand geometry at purdue university's recreational center: an analysis of user perspectives and system performance. Security Technology, 2005. CCST '05. 39th Annual 2005 International Carnahan Conference on, 11-14, pp. 83-88.

[15] Kukula, E. and Elliott, S. (2006) Implementation of hand geometry: an analysis of user perspectives and system performance. Aerospace and Electronic Systems Magazine, IEEE, 21, 3-9.

[16] Ijiri, Y., Sakuragi, M., and Lao, S. (2006) Security management for mobile devices by face recognition. Mobile Data Management, 2006. MDM 2006. 7th International Conference on, 10-12, pp. 49 - 49.

[17] Abeni, P., Baltatu, M., and D’Alessandro, R. (2006) Nis03-4: Implementing biometrics-based authentication for mobile devices. Global Telecommunications Conference, 2006. GLOBECOM 'O6. IEEE, nov., pp. $1-5$.

[18] Shabeer, H. and Suganthi, P. (2007) Mobile phones security using biometrics. Conference on Computational Intelligence and Multimedia Applications, 2007. International Conference on, 13-15, pp. $270-274$.

[19] Saevanee, H. and Bhatarakosol, P. (2008) User authentication using combination of behavioral biometrics over the touchpad acting like touch screen of mobile device. Computer and Electrical Engineering, 2008. ICCEE 2008. International Conference on, 20-22, pp. $82-86$.

[20] Han, Y., Tan, T., Sun, Z., and Hao, Y. (2007) Embedded palmprint recognition system on mobile devices. ICB, pp. 1184-1193. 
[21] McLoughlin, I. and Naidu, N. (2009) Keypress biometrics for user validation in mobile consumer devices. Consumer Electronics, 2009. ISCE '09. IEEE 13th International Symposium on, 25-28, pp. $280-284$.

[22] Kumar, A. and Zhang, D. (2007) Hand-geometry recognition using entropy-based discretization. Information Forensics and Security, IEEE Transactions on, 2, 181-187.

[23] Gross, R., Li, Y., Sweeney, L., Jiang, X., Xu, W., and Yurovsky, D. (2007) Robust hand geometry measurements for person identification using active appearance models. Biometrics: Theory, Applications, and Systems, 2007. BTAS 2007. First IEEE International Conference on, 27-29, pp. 1-6.

[24] Morales, A., Ferrer, M., Alonso, J., and Travieso, C. (2008) Comparing infrared and visible illumination for contactless hand based biometric scheme. Security Technology, 2008. ICCST 2008. 42nd Annual IEEE International Carnahan Conference on, oct., pp. $191-197$.

[25] Rahman, A., Anwar, F., and Azad, S. (2008) A simple and effective technique for human verification with hand geometry. Computer and Communication Engineering, 2008. ICCCE 2008. International Conference on, 13-15, pp. 1177-1180.

[26] Doublet, J., Lepetit, O., and Revenu, M. (2007) Contactless hand recognition based on distribution estimation. Biometrics Symposium, 2007, sept., pp. $1-6$.

[27] Kanhangad, V., Kumar, A., and Zhang, D. (2009) Combining 2d and 3d hand geometry features for biometric verification. Computer Vision and Pattern Recognition Workshops, 2009. CVPR Workshops 2009. IEEE Computer Society Conference on, 20-25, pp. 39-44.

[28] Wang, W.-C., Chen, W.-S., and Shih, S.-W. (2009) Biometric recognition by fusing palmprint and hand-geometry based on morphology. Acoustics, Speech and Signal Processing, 2009. ICASSP 2009. IEEE International Conference on, april, pp. $893-896$.

[29] Michael, G., Connie, T., Hoe, L. S., and Jin, A. (2010) Locating geometrical descriptors for hand biometrics in a contactless environment. Information Technology (ITSim), 2010 International Symposium in, june, pp. $1-6$.

[30] Kanhangad, V., Kumar, A., and Zhang, D. (2010) Human hand identification with $3 \mathrm{~d}$ hand pose variations. Computer Vision and Pattern Recognition Workshops (CVPRW), 2010 IEEE Computer Society Conference on, june, pp. $17-21$.

[31] Alpert, S., Galun, M., Brandt, A., and Basri, R. (2011) Image segmentation by probabilistic bottom-up aggregation and cue integration. Pattern Analysis and Machine Intelligence, IEEE Transactions on, PP, 1.

[32] Munoz, A. G.-C., de Santos Sierra, A., Avila, C. S., Casanova, J. G., del Pozo, G. B., and Vera, V. J. (2010) Hand biometric segmentation by means of fuzzy multiscale aggregation for mobile devices, . aug., pp. 1 -6 .

[33] Sanchez-Reillo, R., Sanchez-Avila, C., and Gonzalez-Marcos, A. (2000) Biometric identification through hand geometry measurements. Pattern Analysis and Machine Intelligence, IEEE Transactions on, 22, 11681171.

[34] Boreki, G. and Zimmer, A. (2005) Hand geometry: a new approach for feature extraction. Automatic Identification Advanced Technologies, 2005. Fourth IEEE Workshop on, 17-18, pp. 149-154.

[35] Kumar, A. (2008) Incorporating cohort information for reliable palmprint authentication. Proc. ICVGIP, Bhubneshwar, India, , Dec, pp. 583590.

[36] Kumar, A., Wong, D., Shen, H., and Jain, A. (2003) Personal verification using palmprint and hand geometry biometrics. Proceedings of the fourth International Conference on audio- and video-based biometric personal authentication. 